\title{
Elevation of serum CXCL16 level correlates well with atherosclerotic ischemic stroke
}

\author{
Aijun $\mathrm{Ma}^{1}$, Xudong Pan ${ }^{1}$, Yubo Xing ${ }^{2}$, Mei Wu ${ }^{3}$, Yuan Wang ${ }^{1}$, Cuiling $\mathrm{Ma}^{1}$
}

${ }^{1}$ Department of Neurology, the Affiliated Hospital of the Medical College, Qingdao University, Qingdao, PR China

2Department of Neurology, Haiyang Hospital, Haiyang, PR China

${ }^{3}$ Laboratory of Human Micromorphology, The Medical College of Qingdao University,

Qingdao, PR China

Submitted: 1 December 2012

Accepted: 1 March 2013

Arch Med Sci 2014; 10, 1: 47-52

DOI: 10.5114 /aoms.2013.39200

Copyright $\odot 2014$ Termedia \& Banach

\section{Abstract}

Introduction: Currently there are no reliable biological markers for ischemic stroke. The novel chemokine CXCL16 is known to be involved in the development of atherosclerosis. Nevertheless, the real role of CXCL16 in atherosclerotic disorders remains uncertain. The goal of our study was to investigate the associations between serum-soluble CXCL16 level and atherosclerotic ischemic stroke, including large artery atherosclerosis (LAA) and small artery occlusion (SAO) subtypes, and to explore whether elevation in CXCL16 levels is correlated with the severity of large arterial stenosis.

Material and methods: The study recruited 227 subjects, including 74 controls and 153 consecutive patients with acute ischemic stroke from atherosclerosis of the carotid artery. The etiology of the acute ischemic strokes was classified into LAA $(n=86)$ subtype and SAO $(n=67)$ subtype according to the TOAST criteria, and the severity of carotid artery stenosis was assessed by the NASCET criteria. Serum-soluble CXCL16 concentration was measured by enzyme-linked immunosorbent assay.

Results: Serum CXCL16 concentrations were significantly increased in both LAA $(2.36 \mathrm{ng} / \mathrm{ml})$ and SAO subtypes $(2.13 \mathrm{ng} / \mathrm{ml})$ when compared to that of the controls ( $2.04 \mathrm{ng} / \mathrm{ml}, p<0.01$ and $p<0.05$, respectively), and it was significantly elevated in LAA subtype than in SAO subtype $(p<0.05)$. However, significant differences in CXCL16 levels between the high-grade stenosis group $(2.36 \mathrm{ng} / \mathrm{ml})$ and moderate-grade stenosis group $(2.24 \mathrm{ng} / \mathrm{ml})$ of LAA subtype were not found $(p>0.05)$. A correlation of serum levels of CXCL16 with serum levels of hs-CRP, fibrinogen and lipid parameters was not observed $(p>0.05)$.

Conclusions: Increased serum level of soluble CXCL16 was independently associated with atherosclerotic ischemic stroke, particularly LAA subtype.

Key words: CXCL16, chemokine, atherosclerosis, ischemic stroke, inflammation.

\section{Introduction}

Stroke is known to be one of the major causes of mortality worldwide and the leading cause of long-term disability as well [1]. In China, ischemic stroke accounts for more than $80 \%$ of all strokes [2]. Clinically ischemic stroke is mainly caused by atherosclerosis, which was once considered to be due to the deposition of lipids within the vascular wall of large or
Corresponding author: Xudong Pan

Department of Neurology the Affiliated Hospital of the Medical College Qingdao University No. 59 Haier Road Qingdao, Shandong Province 266100, PR China Phone: +8653282913033 Fax: +86 53282911840 E-mail: maaijun.cn@163.com 
medium-sized arteries. However, growing evidence has shown that atherosclerosis was not only a process of lipid accumulation but also an inflammatory outcome [3]. In fact, inflammation, together with endothelial dysfunction, played key roles in the initiation and progression of atherosclerosis leading to stroke, myocardial infarction, and other thrombo-occlusive diseases $[4,5]$. During the inflammatory process, chemokines, scavenger receptors and adhesion molecules were all activated [6].

CXCL16, also named SR-PSOX (scavenger receptor that binds phosphatidylserine and oxidized lipoprotein), was a recently discovered chemokine with functions of scavenger receptors and chemotactic properties [7, 8]. It was observed that there was high expression of CXCL16/SR-PSOX mRNA and protein in plaques of coronary and carotid atherosclerosis, and CXCL16 has been proposed to act as a pathogenic mediator in atherosclerosis [9-12]. CXCL16 acted as a scavenger receptor to internalize the oxidized low-density lipoprotein (ox-LDL), and was present in lipid-laden macrophages and atherosclerotic lesions $[7,9,13]$. CXCL16 also existed in a soluble form caused by the metalloproteinase ADAM10 cleavage, and interacted with its specific chemokine receptor designated as CXCR6 to guide the migration of activated T lymphocytes into the inflamed tissue $[8,14]$.

However, the relationship between circulating level of soluble CXCL16 and atherosclerotic disorders remains controversial in clinical practice. Both decreased and increased CXCL16 levels have been reported in patients with atherosclerotic disorders $[10,15]$. Furthermore, there have been no reports about the correlation between the serum levels of CXCL16 with the different subtypes of ischemic stroke. Therefore, the goal of the present study was to investigate the serum levels of CXCL16 in patients with acute ischemic stroke, including the large artery atherosclerosis (LAA) and small artery occlusion (SAO) subtypes, so as to explore whether the circulating level of CXCL16 correlates with the severity of carotid artery stenosis. If so, changes in the circulating level of CXCL16 may serve as a potential diagnostic biomarker for atherosclerotic ischemic stroke.

\section{Material and methods}

\section{Subjects}

A total of 227 subjects were enrolled in the present study, including 74 controls and 153 consecutive patients with acute ischemic stroke ( $\leq 3$ days) from atherosclerosis of the carotid artery. The patients were all admitted to the stroke unit in the Affiliated Hospital of Qingdao University. There were 107 men and 46 women aged 39-88 years in the patient group. The control subjects were selected from the Healthcare Clinic, matched for sex and age (39-85 years).
Detailed history inquiry, neurological examination, risk factor assessment, and imaging studies, such as computed tomography (CT) or/ and magnetic resonance imaging (MRI) of the brain, transcranial Doppler (TCD), and carotid duplex ultrasonography were performed among all participants. Computed tomography angiography or MR angiography of the brain arteries was further done for the patients with acute ischemic stroke after hospitalization. Digital subtraction angiography (DSA) was performed among those with unconfirmed status. The degree of carotid artery stenosis was determined by the North American Symptomatic Carotid Endarterectomy Trial (NASCET) criteria [16]. Patients with acute ischemic stroke were further classified into two clinical subtypes, LAA and SAO, according to TOAST criteria (Trial of Org 10172 in Acute Stroke Treatment) [17].

Exclusion criteria for ischemic stroke patients and controls included previous stroke, stroke of other subtypes, peripheral vascular disease, liver or renal insufficiency, systemic inflammation, autoimmune diseases and cancer. The controls had no neurological abnormality and their brain CT or MRI showed no silent brain infarction.

The study was approved by the Institute Ethical Committee, and informed consent to participate in this study was signed by all participants.

\section{Serology sample collection and storage}

Venous blood samples were drawn from the antecubital vein after an overnight fast. For ischemic stroke patients, blood samples were obtained within $24 \mathrm{~h}$ of admission. Isolated by centrifugation at $3000 \times \mathrm{g}$ for $10 \mathrm{~min}$, serum or plasma samples were aliquoted and stored at $-70^{\circ} \mathrm{C}$ until analyzed. All samples were thawed only once.

\section{Laboratory measurements}

Serum-soluble CXCL16 concentration was measured with the human CXCL16 Quantikine ELISA Kit (R\&D Systems, Minneapolis, MN, USA) according to the manufacturer's instructions. Absorbance was measured by a Benchmark Microplate reader (Bio-Rad, Hercules, CA).

Total cholesterol (TC), low-density lipoprotein $(\mathrm{LDL})$, high-density lipoprotein $(\mathrm{HDL})$, and triglycerides (TG) were assessed by routine methods using a fully automatic biochemical analyzer ( $\mathrm{Hi}-$ tachi 7600-020, Hitachi, Tokyo, Japan). Serum high sensitivity C-reactive protein (hs-CRP) was measured by immunoturbidimetric assay with the automatic biochemical analyzer. Plasma fibrinogen (Fg) was measured by the Von Clauss coagulative method (Fibri-Prest Automate, Diagnostica Stago, Asnieres, France). 


\section{Statistical analysis}

Statistical analyses were performed using the SPSS 11.5 for Windows software (SPSS Inc., Chicago, IL). The Kolmogorov-Smirnov test was used to evaluate whether each variable followed a normal distribution. Normally distributed variables were presented as mean \pm standard deviation (SD), and non-normally distributed variables as medians with inter-quartile ranges. Student's $t$ test or Mann-Whitney $U$ test was used to test for differences between two groups. One-way analysis of variance (ANOVA) followed by the Student-Newman-Keuls test or Kruskal-Wallis test was used to test for differences among several groups. For the enumeration data, the $\chi^{2}$ test was used to compare means. Spearman rank correlation coefficient was used to assess the relationship between serum CXCL16 concentration and other parameters. A $p$ value less than 0.05 was considered statistically significant.

\section{Results}

\section{Demographic profile}

Of all stroke patients, there were 86 patients with LAA stroke, and 67 with SAO stroke according to TOAST criteria. There were no statistical differences in age $(F=1.11, p=0.33)$, gender $\left(\chi^{2}=2.55, p=0.28\right)$, active smoking $\left(\chi^{2}=1.31, p=\right.$ $0.52)$ or alcohol abuse $\left(\chi^{2}=2.68, p=0.26\right)$ among the participants. Differences in co-existence with coronary artery disease (CAD, $\left.\chi^{2}=0.57, p=0.75\right)$, hypertension $\left(\chi^{2}=5.54, p=0.06\right)$ and diabetes $\left(\chi^{2}=1.39, p=0.5\right)$ were not significant either among these three groups (Table I).

\section{Laboratory results}

Laboratory findings are presented in Figure 1 and Table II. Serum CXCL16 concentrations were significantly increased in LAA and SAO stroke pa- tients compared to those of the controls $(p<0.01$, $p<0.05)$, and were also significantly higher in LAA subtype than in SAO subtype ( $p<0.05$, Figure 1$)$.

Serum hs-CRP and Fg concentrations were significantly increased in both LAA and SAO compared to those of controls ( $p<0.01$ ), and the concentration of hs-CRP was higher in LAA subtype than in SAO subtype $(p<0.01$, Table II).

Serum levels of TG and LDLs were much higher in both LAA and SAO compared to those of controls ( $p<0.01, p<0.05$, Table II), but significant differences between the two subtypes were not found. There were no significant differences in TC or HDL levels among the three groups.

\section{Relationships of serum CXCL16 concentration to plasma lipoproteins, hs-CRP and Fg}

Serum CXCL16 concentration had no correlation with age or gender. Furthermore, serum

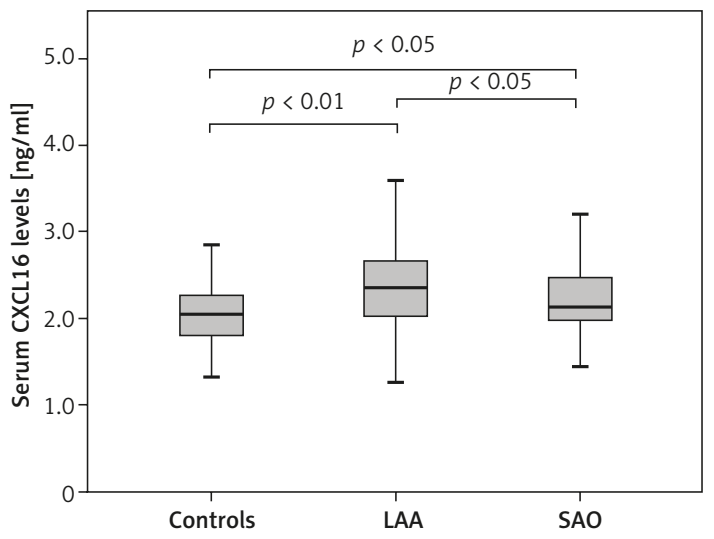

Figure 1. Serum CXCL16 levels in controls, patients with LAA and SAO stroke. Horizontal line in the box plots represents the median value; the boxed area is the interquartile range and the whiskers denote the minimum and the maximum

Table I. Demographic characteristics of ischemic stroke patients and controls

\begin{tabular}{|llll|}
\hline Variables & Controls $(n=74)$ & \multicolumn{2}{c|}{ Ischemic stroke } \\
\cline { 3 - 4 } & & LAA $(n=86)$ & SAO $(n=67)$ \\
\hline Age [years] & $65.96 \pm 11.54$ & $68.29 \pm 11.47$ & $65.93 \pm 11.48$ \\
\hline \begin{tabular}{l} 
Gender, $n(\%)$ \\
\hline Male
\end{tabular} & $44(59.5)$ & $61(70.9)$ & $46(68.7)$ \\
\hline Female & $30(40.5)$ & $25(29.1)$ & $21(31.3)$ \\
\hline Active smoker, $n(\%)$ & $26(35.1)$ & $27(31.4)$ & $27(40.3)$ \\
\hline Alcohol abuse, $n(\%)$ & $16(21.6)$ & $27(31.4)$ & $22(32.8)$ \\
\hline Hypertension, $n(\%)$ & $42(56.7)$ & $64(74.4)$ & $24(65.7)$ \\
\hline Diabetes, $n(\%)$ & $19(25.7)$ & $29(33.7)$ & $21(32.8)$ \\
\hline CAD, $n(\%)$ & $19(25.7)$ & $25(29.1)$ & \\
\hline
\end{tabular}


Table II. Clinical laboratory findings of ischemic stroke patients and controls

\begin{tabular}{|c|c|c|c|}
\hline \multirow[t]{2}{*}{ Variables } & \multirow[t]{2}{*}{ Controls $(n=74)$} & \multicolumn{2}{|c|}{ Ischemic stroke } \\
\hline & & LAA $(n=86)$ & SAO $(n=67)$ \\
\hline CXCL16 [ng/ml] & $2.04(1.80,2.26)$ & $2.36(2.03,2.68)^{\star, \#}$ & $2.13(1.92,2.50)^{\Delta}$ \\
\hline hs-CRP [mmol/l] & $1.13(0.65,1.69)$ & $3.43(1.55,10.47)^{\star,+}$ & $1.41(0.93,3.85)^{\star}$ \\
\hline $\mathrm{Fg}[\mathrm{g} / \mathrm{l}]$ & $2.40(1.80,2.26)$ & $3.14(2.51,3.70)^{\star}$ & $2.74(2.56,3.40)^{\star}$ \\
\hline $\mathrm{TC}[\mathrm{mmol} / \mathrm{l}]$ & $4.51 \pm 1.00$ & $4.89 \pm 1.19$ & $4.80 \pm 1.13$ \\
\hline $\mathrm{TG}[\mathrm{mmol} / \mathrm{l}]$ & $1.42 \pm 0.37$ & $1.78 \pm 0.59^{\star}$ & $1.63 \pm 0.49^{\Delta}$ \\
\hline LDL [mmol/l] & $2.49 \pm 0.70$ & $2.85 \pm 0.92^{*}$ & $2.85 \pm 0.83^{*}$ \\
\hline HDL [mmol/l] & $1.31 \pm 0.31$ & $1.24 \pm 0.24$ & $1.27 \pm 0.28$ \\
\hline
\end{tabular}

CXCL16 concentration had no correlation with the levels of lipid, lipoprotein fractions, hs-CRP or Fg $(p>0.05)$.

\section{Relationships of serum CXCL16 concentration to the severity of carotid artery stenosis in LAA subtype}

In LAA subtype, 55 patients had high-grade carotid artery stenosis (70-99\%) or occlusion, and the other 31 had a moderate degree of stenosis (50-69\%). The serum CXCL16 concentration in the high-grade stenosis group $(2.36(2.06,2.64) \mathrm{ng} / \mathrm{ml})$ was higher than that in the moderate-grade stenosis group $(2.24(2.02,2.71) \mathrm{ng} / \mathrm{ml})$, but the difference between them was not statistically significant $(p>0.05)$.

\section{Discussion}

The present study showed that circulating CXCL16 levels in patients with acute ischemic stroke from atherosclerosis of the carotid artery, especially in LAA subtype, were significantly increased when compared to those of the controls. Furthermore, the elevation of CXCL16 levels was not found to be associated with other acute phase reactants or serum lipid parameters. Therefore, it is likely that systematic serum CXCL16 level might be independently associated with the development of atherosclerotic ischemic stroke. Similar to our result, Smith et al. reported increased plasma levels of CXCL16 in coronary artery disease (CAD) independent of co-morbidity such as diabetes and hypertension [10]. They found that soluble CXCL16 induced inflammatory responses in vascular smooth muscle cells (SMC) and human peripheral blood mononuclear cells (PBMCs), with particularly prominent effects on PBMCs in CAD patients, which suggested that soluble CXCL16 could be linked to atherogenesis not only as a marker of inflammation, but also as a potential inflammatory mediator.
There is increasing evidence illustrating that inflammation plays an important role in the pathophysiology of ischemic stroke [18-20]. Atherosclerosis is a complex disease which involves chronic inflammation at every stage, from initiation to progression and eventually to plaque rupture, and some inflammatory markers are even related to vulnerable plaque stabilization and the prognosis of thrombo-occlusive diseases [4, 21]. Consistent with these data, levels of acute inflammatory reactants, such as hs-CRP and Fg, were also found significantly increased in patients with acute ischemic stroke in our study.

Based on its potential action as a scavenging receptor internalizing ox-LDL and showing enhanced expression in atherosclerotic plaques [7, 9, 11], CXCL16 was thought to play a crucial role in pathogenesis of atherosclerosis. It was reported that CXCL16 interacts with its specific chemokine receptor CXCR6 to guide the migration of activated $T$ cells into inflamed tissue [8, 13], and activated $T$ cell begins producing tumor necrosis factor (TNF), interleukin-2, etc, which are involved in atherosclerosis. Upon studying the CXCL16 expression in human mononuclear cells, SMC and endothelial cells (ECs), it was found that CXCL16 mRNA could also be induced by interferon (IFN)- $\gamma$ and TNF- $\alpha[13,22]$. Interferon- $\gamma$ treatment of human PBMCs, THP-1 cells and SMCs promotes the uptake of ox-LDL by these cells, which may be mediated by increased expression of membrane-bound CXCL16. On the other hand, anti-CXCL16 antibodies could block the uptake of ox-LDL by human PBMCs in vitro $[11,23]$. Therefore, CXCL16 is a link between pro-inflammatory stimuli and lipid uptake [6]. Our study suggested that soluble CXCL16 levels were independently associated with atherosclerotic ischemic stroke, and particularly LAA subtype. Higher CXCL16 levels in LAA subtype than in SAO subtype may suggest that metabolic dyslipidemia and inflam- 
matory risk factors may contribute more to the former process.

The relationship of serum CXCL16 concentration to severity of artery stenosis was debatable. Circulating CXCL16 could or could not be related to the severity of coronary artery stenosis [24, 25]. Wang et al. [26] suggested that serum CXCL16 concentration was strongly associated with increased carotid intima-media thickness and vulnerable plaque. Our study suggested that serum CXCL16 level was not linked to the severity of carotid artery stenosis in LAA stroke patients. The explanation of this discrepancy is that vulnerable plaque contributes more to the process of atherothrombotic stroke or embolism than artery stenosis itself, and there is probably no association between the vulnerability of a plaque and the severity of artery stenosis. CXCL16 induces angiogenesis as a vascular-derived factor [27], and the neovascularization is a core event in the rupture of plaques [28].

The results of the study are limited to some degree since it is difficult to study the status of membrane-bound CXCL16 and other inflammatory parameters in our patients. Such knowledge may help clarify the exact role of CXCL16 and its association with the development of atherosclerotic ischemic stroke. Nevertheless, our study has revealed that increased levels of serum-soluble CXCL16 were independently associated with the presence of atherosclerotic ischemic stroke. More studies are needed to further elucidate the role of CXCL16 as a biomarker of atherosclerotic stroke that can be applied in clinical practice or stroke epidemiology studies.

\section{Acknowledgments}

Aijun Ma and Xudong Pan - equal contributors. The authors would like to thank Dr. Guirong Sun for excellent technical assistance. Dr. Xudong Pan, the corresponding author, was partially supported by a grant from Shandong Province Natural Science Foundation (ZR2011HM087).

\section{References}

1. Chalela JA, Merino JG, Warach S. Update on stroke.Curr Opin Neurol 2004; 17: 447-51.

2. Zhao D, Liu J, Wang W, et al. Epidemiological transition of stroke in China: twenty-one-year observational study from the Sino-MONICA-Beijing Project. Stroke 2008; 39: 1668-74.

3. Flossmann E, Schulz UG, Rothwell PM. Potential confounding by intermediate phenotypes in studies of the genetics of ischaemic stroke. Cerebrovasc Dis 2005; 19: 1-10.

4. Libby P, Ridker PM, Maseri A. Inflammation and atherosclerosis. Circulation 2002; 105: 1135-43.

5. Ross R. Atherosclerosis: an inflammatory disease. N Engl J Med 1999; 340: 115-26.
6. Sheikine Y, Sirsjö A. CXCL16/SR-PSOX: a friend or a foe in atherosclerosis? Atherosclerosis 2008; 197: 487-95.

7. Shimaoka T, Kume N, Minami M, et al. Molecular cloning of a novel scavenger receptor for oxidized low density lipoprotein, SR-PSOX, on macrophages. J Biol Chem 2000; 275: 40663-6.

8. Matloubian M, David A, Engel S, Ryan JE, Cyster JG. A transmembrane CXC chemokine is a ligand for HIVcoreceptor Bonzo. Nat Immunol 2000; 1: 298-304.

9. Minami M, Kume N, Shimaoka T, et al. Expression of scavenger receptor for phosphatidylserine and oxidized lipoprotein (SR-PSOX) in human atheroma. Ann N Y Acad Sci 2001; 947: 373-6.

10. Smith C, Halvorsen B, Otterdal K, et al. High levels and inflammatory effects of soluble CXC ligand 16 (CXCL16) in coronary artery disease: down-regulatory effects of statins. Cardiovasc Res 2008; 79: 195-203.

11. Wuttge DM, Zhou X, Sheikine Y, et al. CXCL16/SR-PSOX is an interferon-gamma-regulated chemokine and scavenger receptor expressed in atherosclerotic lesions. Arterioscler Thromb Vasc Biol 2004; 24: 750-5.

12. Minami M, Kume N, Shimaoka T, et al. Expression of SR-PSOX, a novel cell-surface scavenger receptor for phosphatidylserine and oxidized LDL in human atherosclerotic lesions. Arterioscler Thromb Vasc Biol 2001; 21: 1796-800.

13. Aslanian AM, Charo IF. Targeted disruption of the scavenger receptor and chemokine CXCL16 accelerates atherosclerosis. Circulation 2006; 114: 583-90.

14. Abel S, Hundhausen C, Mentlein R, et al. The transmembrane CXC-chemokine ligand 16 is induced by IFN-gam$\mathrm{ma}$ and TNF-alpha and shed by the activity of the disintegrin-like metalloproteinase ADAM10. J Immunol 2004; 172: 6362-72.

15. Sheikine Y, Bang CS, Nilsson L, et al. Decreased plasma CXCL16/SR-PSOX concentration is associated with coronary artery disease. Atherosclerosis 2006; 188: 462-6.

16. Beneficial effect of carotid endarterectomy in symptomatic patients with high-grade carotid stenosis. North American Symptomatic Carotid Endarterectomy Trial Collaborators. N Engl J Med 1991; 325: 445-53.

17. Adams HP Jr, Bendixen BH, Kappelle LJ, et al. Classification of subtype of acute ischemic stroke. Definitions for use in a multicenter clinical trial. TOAST. Trial of Org 10172 in Acute Stroke Treatment. Stroke 1993; 24: 35-41.

18. Waje-Andreassen U, Kråkenes J, Ulvestad E, et al. IL-6: an early marker for outcome in acute ischemic stroke. Acta Neurol Scand 2005; 111: 360-5.

19. Idicula TT, Brogger J, Naess H, Waje-Andreassen U, Thomassen L. Admission C-reactive protein after acute ischemic stroke is associated with stroke severity and mortality: the 'Bergen stroke study'. BMC Neurol 2009; 9: 18.

20. Di Napoli M, Singh P. Is plasma fibrinogen useful in evaluating ischemic stroke patients: why, how, and when? Stroke 2009; 40: 1549-52.

21. Yu DQ, Lin SG, Chen JY, et al. Effect of atorvastatin therapy on borderline vulnerable lesions in patients with acute coronary syndrome. Arch Med Sci 2011; 7: 433-9.

22. Nakayama T, Hieshima K, Izawa D, Tatsumi Y, Kanamaru A, Yoshie O. Cutting edge: profile of chemokine receptor expression on human plasma cells accounts for their efficient recruitment to target tissues. J Immunol 2003; 170: 1136-40.

23. Wågsäter D, Olofsson PS, Norgren L, Stenberg B, Sirsjö A. The chemokine and scavenger receptor CXCL16/SR-PSOX is expressed in human vascular smooth muscle cells 
and is induced by interferon gamma. Biochem Biophys Res Commun 2004; 325: 1187-93.

24. Sun Y, Chang Z, Zhang S. Increased serum CXCL16 level is a marker for acute coronary syndromes. Arch Med Res 2008; 39: 332-7.

25. Yi GW, Zeng QT. Circulating CXCL16 is related to the severity of coronary artery stenosis. Arch Med Res 2008; 39: 531-5.

26. Wang KD, Liu ZZ, Wang RM, et al. Chemokine CXC Ligand 16 serum concentration but not A181V genotype is associated with atherosclerotic stroke. Clin Chim Acta 2010; 411: 1447-51.

27. Zhuge X, Murayama T, Arai H, et al. CXCL16 is a novel angiogenic factor for human umbilical vein endothelial cells. Biochem Biophys Res Commun 2005; 331: 1295-300.

28. Russell DA, Abbott CR, Gough MJ. Vascular endothelial growth factor is associated with histological instability of carotid plaques. Br J Surg 2008; 95: 576-81. 